\title{
ANALISIS DEL ESTADO DE ESFUERZOS EN LA ZONA DE LA CRUZ CORTINA-DIQUE TALCONAPAN, PUE.
}

José Rafael Aranda López* Antonio Uribe Carbajal*

\section{RES U MEN}

Se presentan los resultados obtenidos de los estudios geofísicos de refracción y sísmica en pozos del tipo "up-hole", realizados en la zona dique cortina Talconapan del río Atexcaco, Pue., asi como los problemas de estabilidad que pudieran presentarse si se construye el sistema hidroeléctrico formado por el dique de $13 \mathrm{~m}$. de altura y una cortina de $40 \mathrm{~m}$, de alturas aproximadas en los materiales vulcanoclásticos del área.

Los modelos de velocidades del perfil obtenidos a partir del método geofisico de "up-hole" exhiben una correlación litoestratigráfica con los perfiles de velocidad de intervalo.

Se muestra un modelo de esfuerzos en dos dimensiones, calculado por medio de elementos finitos. Esto permite determinar zonas de concentración de esfuerzos, los cuales deberán ser tomados en cuenta para el diseño de la obra.

\begin{abstract}
Results of the refraction and sismic up-hole geophysical studies made In Talconapan dam site at Atexcaco river Puebla, are presented.

The velocity profile models obtained from the up-hole method shows litostratigraphic correlation.

This paper presents a two dimensional strain modeling, calculated by finite elements. This allows to determinate strain concentration zones wich must be taken in account for the design.
\end{abstract}

*Comisión Federal de Electricidad. 


\section{INTRODUCCION}

A partir de la Segunda Guerra Mundial la técnica del cálculo de esfuerzos por elementos finitos, ha sido poco a poco adoptada por las diferentes disciplinas de ingeniería, tenlendo mayor aceptación en la aeronáutica y algunas áreas de la ingeniería civil.

Desde mediados de la década de los sesentas se han realizado trabajos en las ciencias de la Tierra, que van desde la determinación de los esfuerzos en obras mineras (Zienkiewicz et al.1966) hasta el cálculo de la probabilidad de ocurrencia de grandes sismos (Uribe y Nyland, 1983).

La determinación de la distribución de esfuerzos en medios anisotrópicos es de gran interés en la mecánica de rocas. Sin embargo, con los métodos clásicos de teoria de elasticidad sólo es posible resolver las geometrias más simples. Por medio de diferencias finitas se puede llegar a resolver problemas donde la anisotropía sea homogénea (Cook, 1974).

El método de elementos finitos en cambio evita formular ecuaciones constitutivas explicitas basándose en consideraciones de energía solamente. A los elementos de un medio continuo se les puede asignar propiedades elásticas a voluntad, según el caso; estas son especificas de cada elemento y pueden tener variaciones según sus características geológicas to cual permite modelar cualquier tipo de anisotropia.

En este estudio se presenta un perfil, en el cual las condiciones geológicas de la cortina-dique Talconapan son analizadas con el fin de determinar la estabilidad de los materiales presentes. Esto se desarrolla utilizando un modelo de elementos finitos en dos dimensiones, y conservando el espesor de todos los elementos fijos.

\section{MARCO GEOLOGICO}

El perfil en cuestión se encuentra ubicado en un sitio cercano a la cludad de Teziutlán, Puebla, en una zona vulcanoclástica (Figura 1) con materiales de mala calidad. Aflora toba arcillo-arenosa deleznable, le subyace un horizonte arcilloso que presenta lentes andesíticos y de toba andesitica soldada, por debajo está la andesita que exhlbe una capa de alteración en su parte superficial (Aranda, 1985).

Sobre la margen derecha de la sección de la cortina, se observa un lente de arena conglomerítico bajo el horizonte arcilloso, cubriendo a la andesita y la toba, (Figura 2 ).

\section{METODOLOGIA GEOFISICA}

En el perfil dique-cortina se realizaron estudlos de refracción sísmica de ondas compresionales y transversales en los pozos III, IV, VI y VII respectivamente.

Los barrenos II y IV están ubicados en el perfil del dique, el barreno $V I$ se encuentra en la margen izquierda de la cortina y el barreno VII en la margen derecha. (Figura 2).

Los datos de tiempo leidos en los sismogramas, se graficaron en diagramas $T-Z$ 
LOCALIZACION DEL AREA DE ESTUDIO

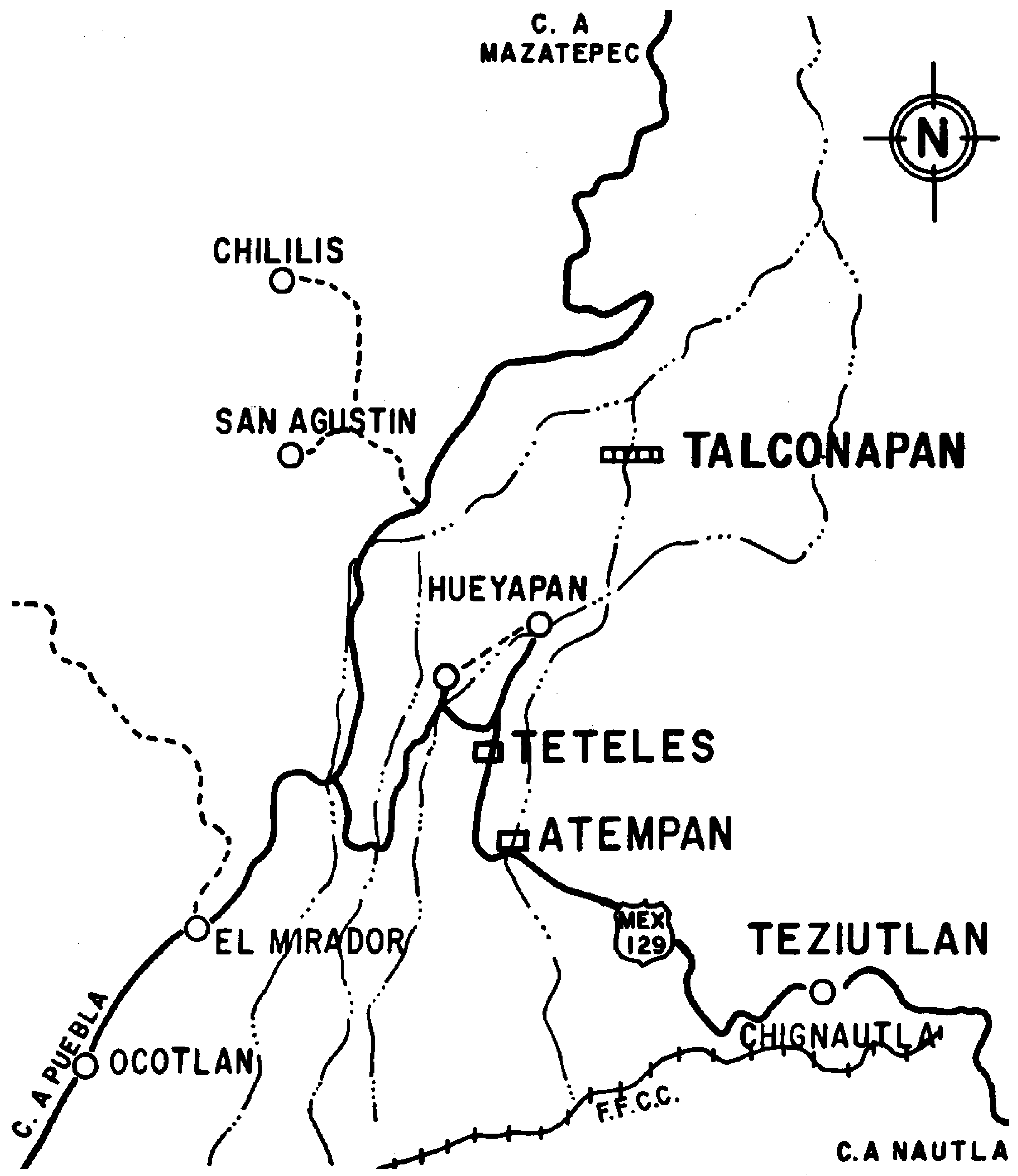

FIGURA 1 


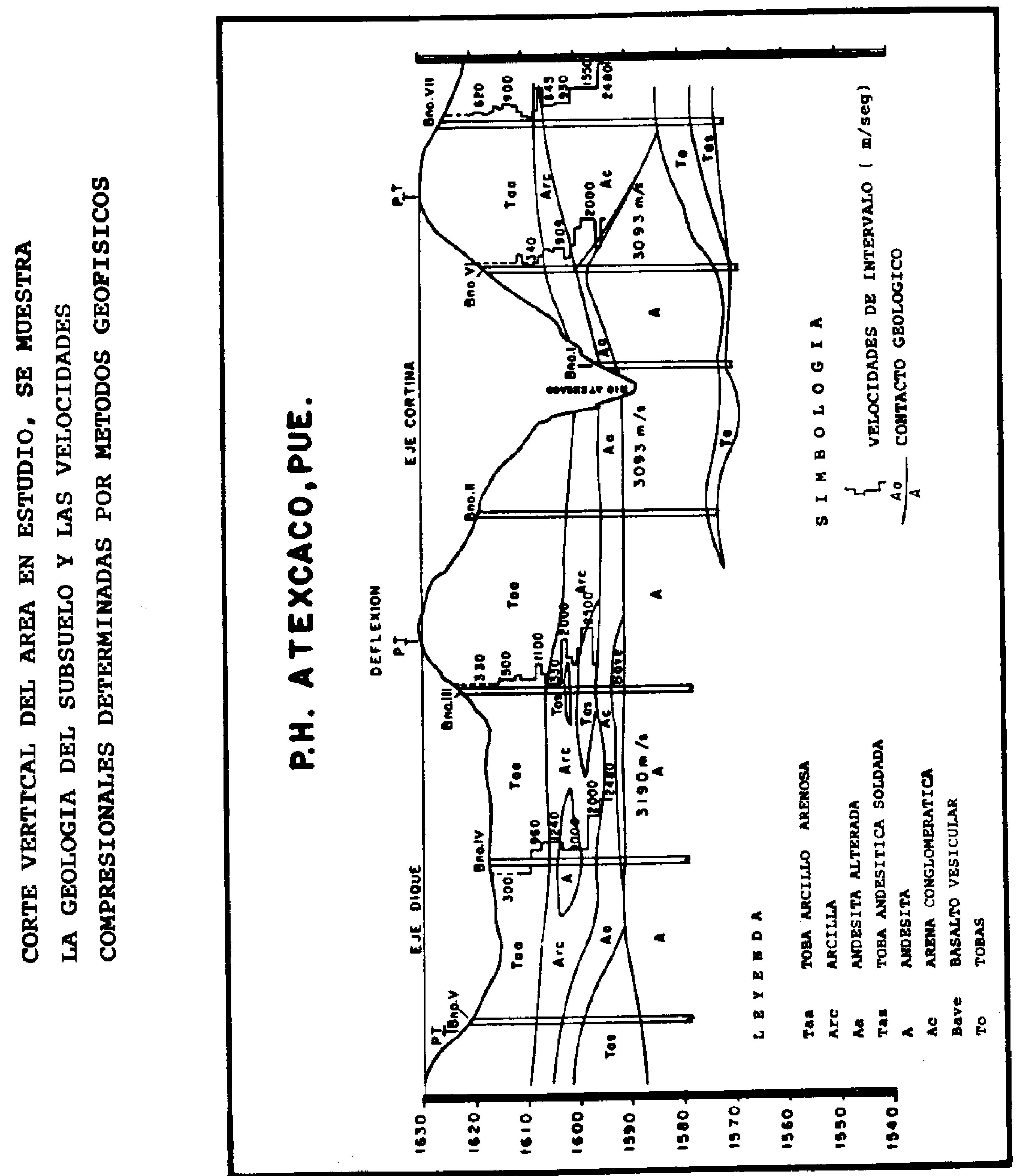

$\frac{1}{6}$ 
(tiempo-profundidad), para obtener las velocidades de onda compresional y transversal de los distintos materiales atravesados hasta profundidades que varian entre 25 y 35 metros (Figura 3). Las velocidades fueron calculadas en forma estadística debido tanto a la calidad como a la cantidad de los arribos de ondas compresional y transversal.

Las densidades de las rocas se dedujeron de los registros geofísicos de densidad. Con estos valores y las velocidades compresional y de corte, se calcularon las constantes elásticas dinámicas por medio de las siguientes expresiones siguiendo a Davenport et al, (1978):

= Np/Ns)-2 Relación de Poisson 2(Vp/Ns)-2

$E=2$ Vsp $\times(1+)$ Módulo de Young

Los valores de velocidad de intervalo graficados sobre el perfil geológico (Figura 2) exhiben una correlación litoestratigráfica y proporciona parámetros de la calidad de los horizontes atravesados.

Sobre la margen derecha se observa una inversión de velocidad en el contacto de la toba arcillo-arenosa con el estrato de arcilla, la cual se considera una zona de inestabilidad. A partir de los módulos de Young y considerando la geología descrita se propone una zonificación preliminar del perfil, (Aranda, J. R., 1985), como lo muestra la Figura 4 se clasifican tres tipos de materiales: el paquete tobáceo superficial, un paquete intermedio arcilloso $y$ un paquete andesítico profundo.

\section{MODELO DE ELEMENTOS FINITO}

Datos

Los datos de módulo de Young, relación de Poisson calculados para cada material según los datos geofísicos, asi como la densidad, se utilizaron para alimentar el programa de elemento finito; como en todas las técnicas es necesario hacer algunas simplificaciones:

Debido a las limitaciones del cálculo y la restricción del programa desarrollado (Uribe y Nyland, 1983), los materiales reales son agrupados de manera que sólo tengan 8 tipos con caracteristicas semejantes considerando la información litológica y geofísica, Tabla 1.

Los materiales 1,2 y 3 corresponden al paquete tobáceo, sus módulos de Young varían entre 0.14 y 0.40 Ton $/ \mathrm{cm} 2$.

El horizonte arcilloso, que tiene parametros elásticos semejantes a los del materlal 2 en la margen derecha del río Atexcaco, mientras que el resto de la sección se clasifican como material 4, con módulo de Young de 0.5 Ton/cm2

El material 5 es la arena conglomerática, tiene un módulo de Young de $1.1 \mathrm{Ton} / \mathrm{cm}$.

El paquete andesítico caracteriza a los materlales 6,7 y 8 , los cuales exhiben módulos de Young de 2.9 a $3.2 \mathrm{Ton} / \mathrm{cm}$.

Todos los parámetros dinámicos para cada uno de los materiales se calcularon a partir de los datos geofísicos que se discutieron con anterioridad.

Considerando los datos goológicos y los parámetros dinámicos calculados, el modelo propuesto se muestra en la Figura 5. 
是
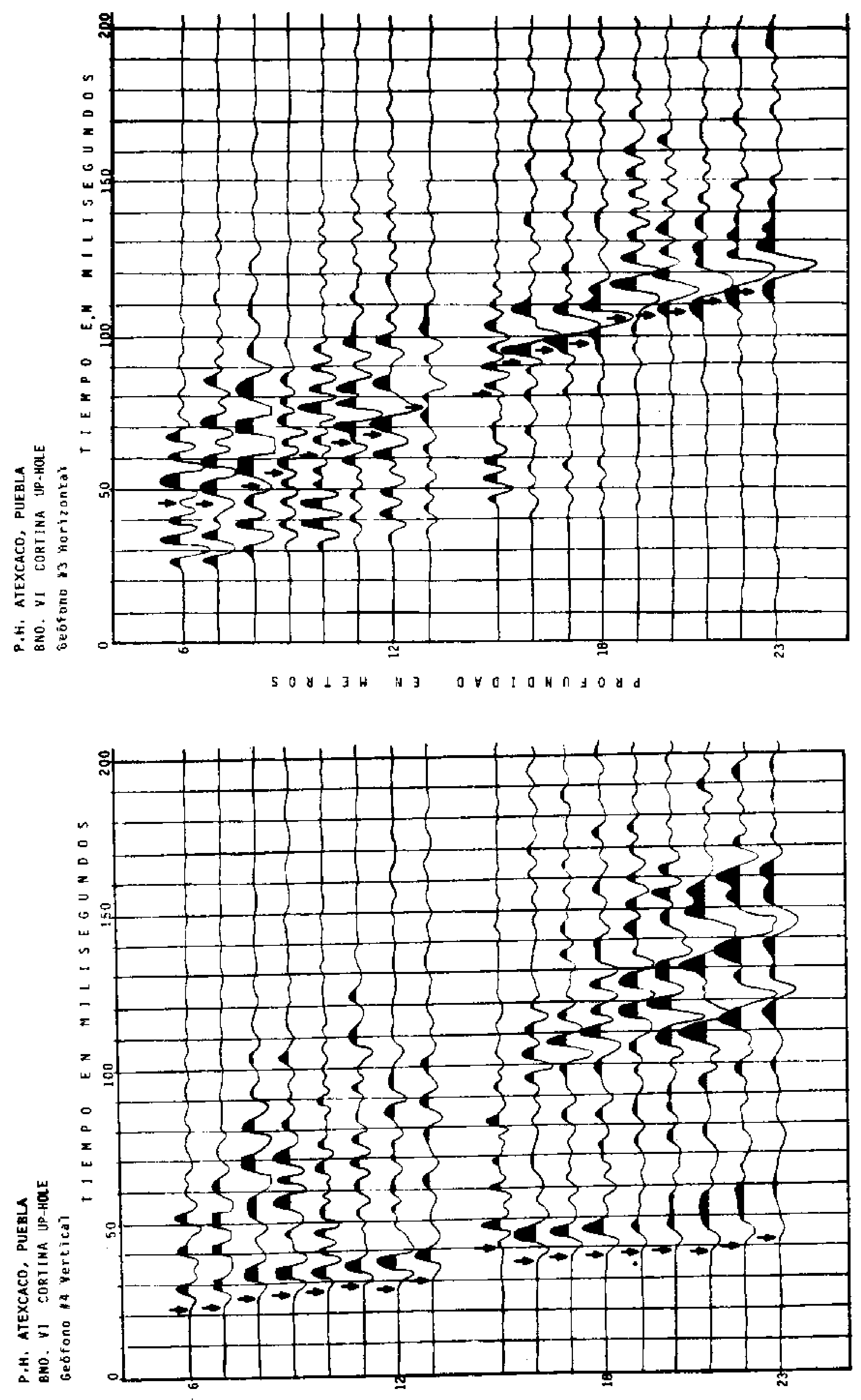

3
0
0 


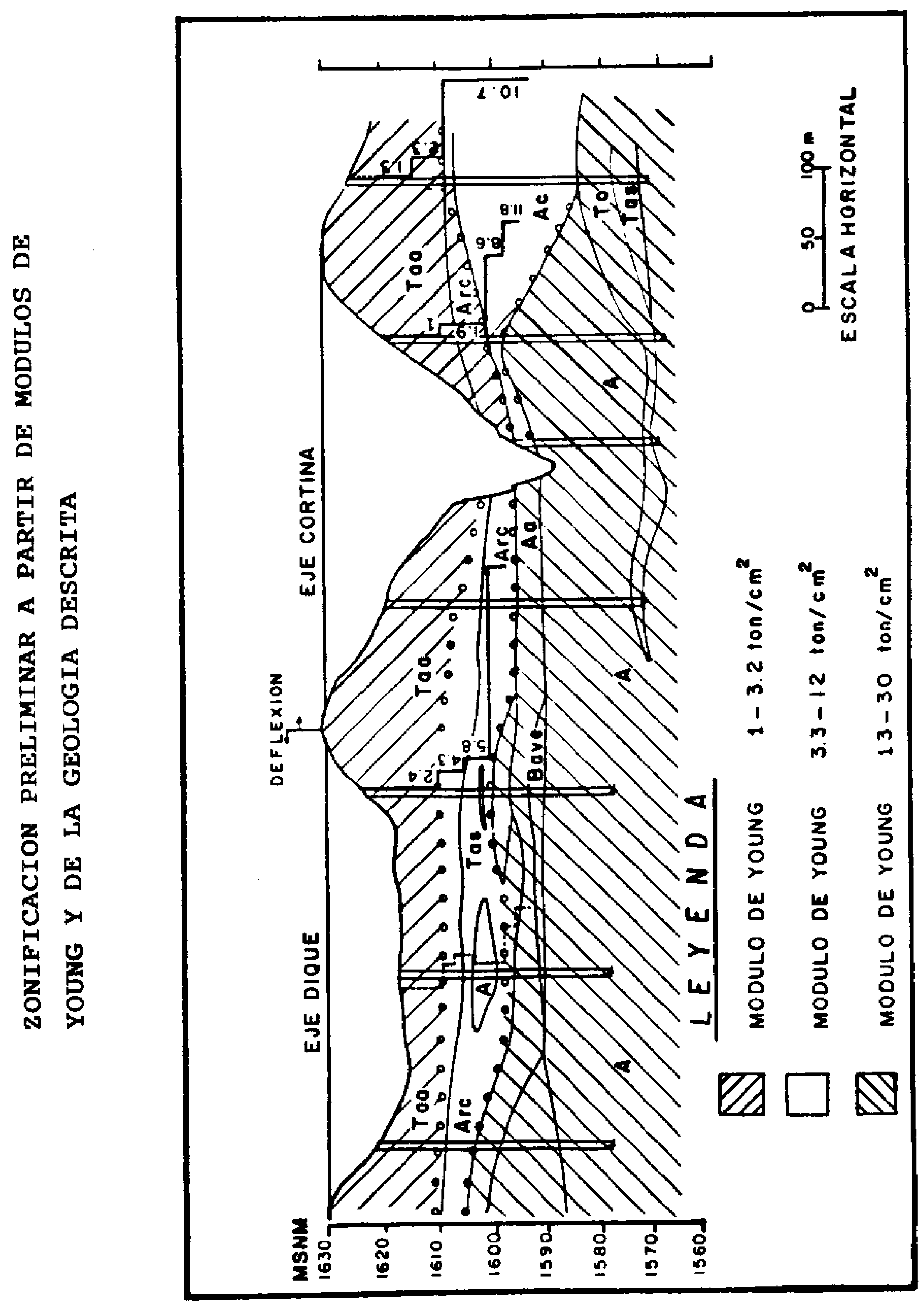


TABLA 1

\begin{tabular}{|c|c|c|c|}
\hline$M A T E R \mid A L$ & $\begin{array}{c}E \\
\left(\operatorname{Ton} / \mathrm{cm}^{2}\right)\end{array}$ & $\mathcal{D}$ & $\begin{array}{c}\gamma \\
\left(g / \mathrm{cm}^{3}\right)\end{array}$ \\
\hline 1 & 0.14 & 0.44 & 1.77 \\
\hline 1217 & 0.24 & 0.44 & 1.77 \\
\hline 3 & 0.40 & 0.39 & 1.77 \\
\hline 4 & 0.50 & 0.38 & 1.8 \\
\hline$E 5 \equiv$ & 1.10 & 0.46 & 2.0 \\
\hline 6 & 1.50 & 0.38 & 2.0 \\
\hline 7 & 2. 90 & 0.44 & 2. 0 \\
\hline 8 & 3.20 & 0.38 & 2.24 \\
\hline
\end{tabular}

P.H. ATEXCACO, PUE.

PARAMETROS ELASTICOS UTILIZADOS EN EL MODELADO

POR ELEMENTOS FINITOS DE LA CORTINA TLALCONALPAN 


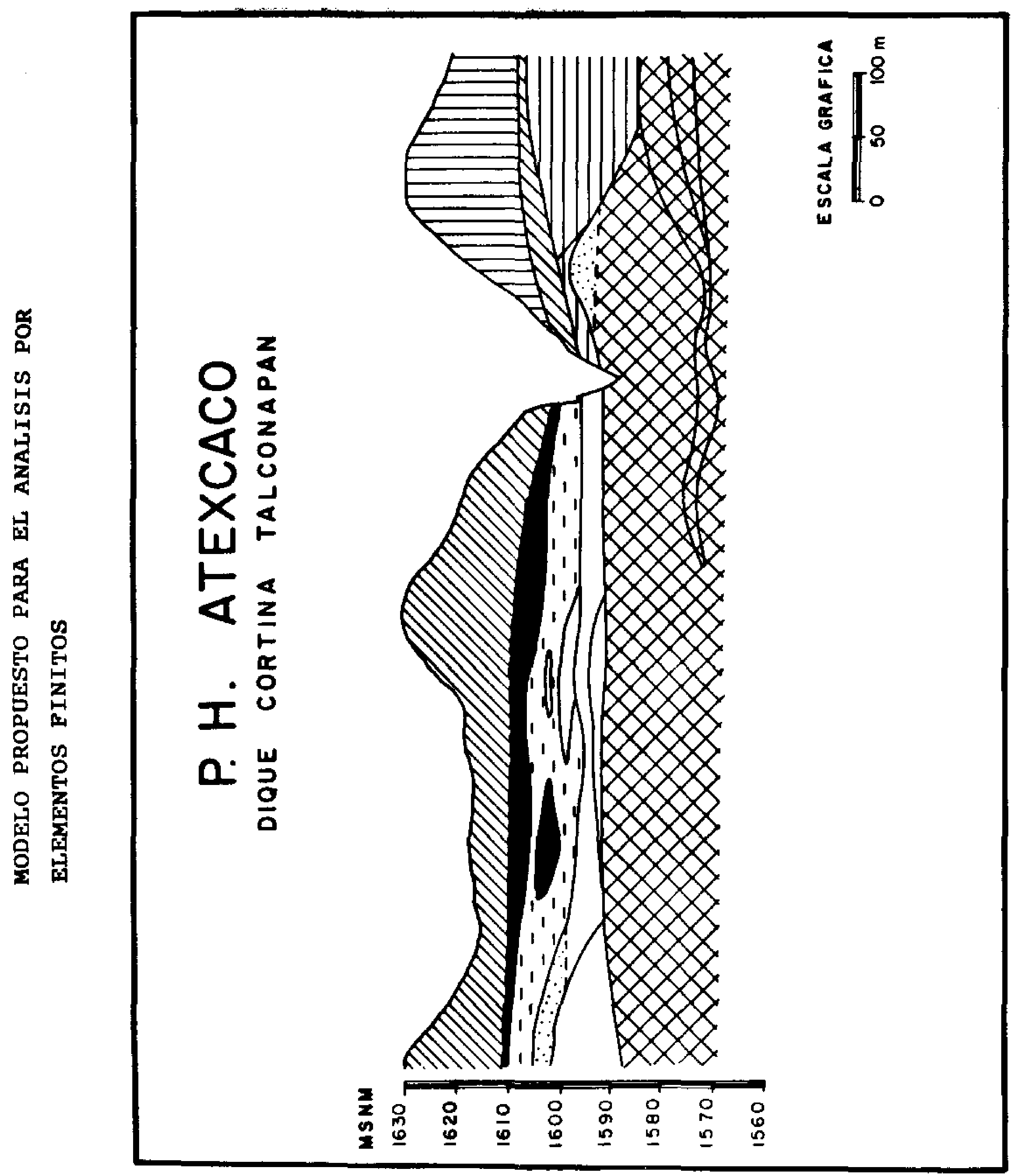

$\frac{n}{6}$ 


\section{MALLA TRIANGULAR}

Todos los materiales se comportan elásticamente considerando una sección transversal en la que los elementos tienen un espesor de $50 \mathrm{~m}$.

Se utilizaron elementos triangulares de esfuerzo constante para construir la malla, donde el tamaño de los elementos va de acuerdo a las características geológicas y de las áreas de mayor interés, como será el caso del vértice de a cañada y la secuencia litológica. También se tomaron on cuenta las variaciones detectadas por el método sísmico de un mismo horizonte (Figura 6) para mayor congruencia del modelo con la realidad.

\section{CONDICIONES INICIALES}

Las condiciones de frontera se han considerado en la base y a los extremos del modelo, éstas permiten desplazamientos verticales en los nodos extremos de la derecha e izquierda para obtener la continuidad hacia el medio circundante y viceversa.

En la base del modelo no se acepta deformación vertical, ésto implica que el área de estudio sobreyace un estrato de mayor rigidez que se asigna como basamento local.

Para determinar la estabilidad del modelo la única fuerza externa que se permite es el efecto gravitacional, pudiendo considerarse en otros casos los esfuerzos producidos por el agua del embalse.

\section{RESULTADOS}

Con esta investigación se determinó el tensor de esfuerzos para cada uno de los nodos del modelo de celdas triangulares.

\section{COMPONENTE HORIZONTAL $(\mathbf{x x})$}

La Flgura 7 exhibe la distribución de la componente $\mathrm{XX}$, ésta presenta la tendencia que tienen los materiales a desplazarse on dirección horizontal. La tendencia térica debe aumentar gradualmente con la profundidad.

En este perfil se observan dos zonas de interés:

La primera se encuentra en la margen izquierda de la cañada que muestra una mayor estabilidad relacionada con la resistencia del material.

La segunda zona de importancia es ta anomalla con valor de $3 E$ O5 dinas $/ \mathrm{cm}$, que se localiza en el centro de la prominencia de la margen derecha lo que implica un área problemática, que también se correlaciona con la anomalía de $340 \mathrm{~m} / \mathrm{seg}$ de velocidad de intervalo, entre los 7 y $10 \mathrm{~m}$ de profundidad, definida por el "hole" del barreno VI (Figura 2).

Esta zona corresponde litológicamente al contacto entre la toba arcillo-arenosa y la capa arcillosa.

\section{COMPONENTE VERTICAL (yy)}

El análisis de la componente vertical correlaciona con el inciso anterior, el área inestable de la toba arcillo-arenosa que se encuentra en margen derecho. (Figura 8).

EI escarpe de la margen izquierda presenta pequeñas anomalías de estabilidad. El resto del perfil exhibe un comportamiento estable dentro de lo inferido respecto a la topografía con excepción del levantamiento de la curva de $7 E$ 


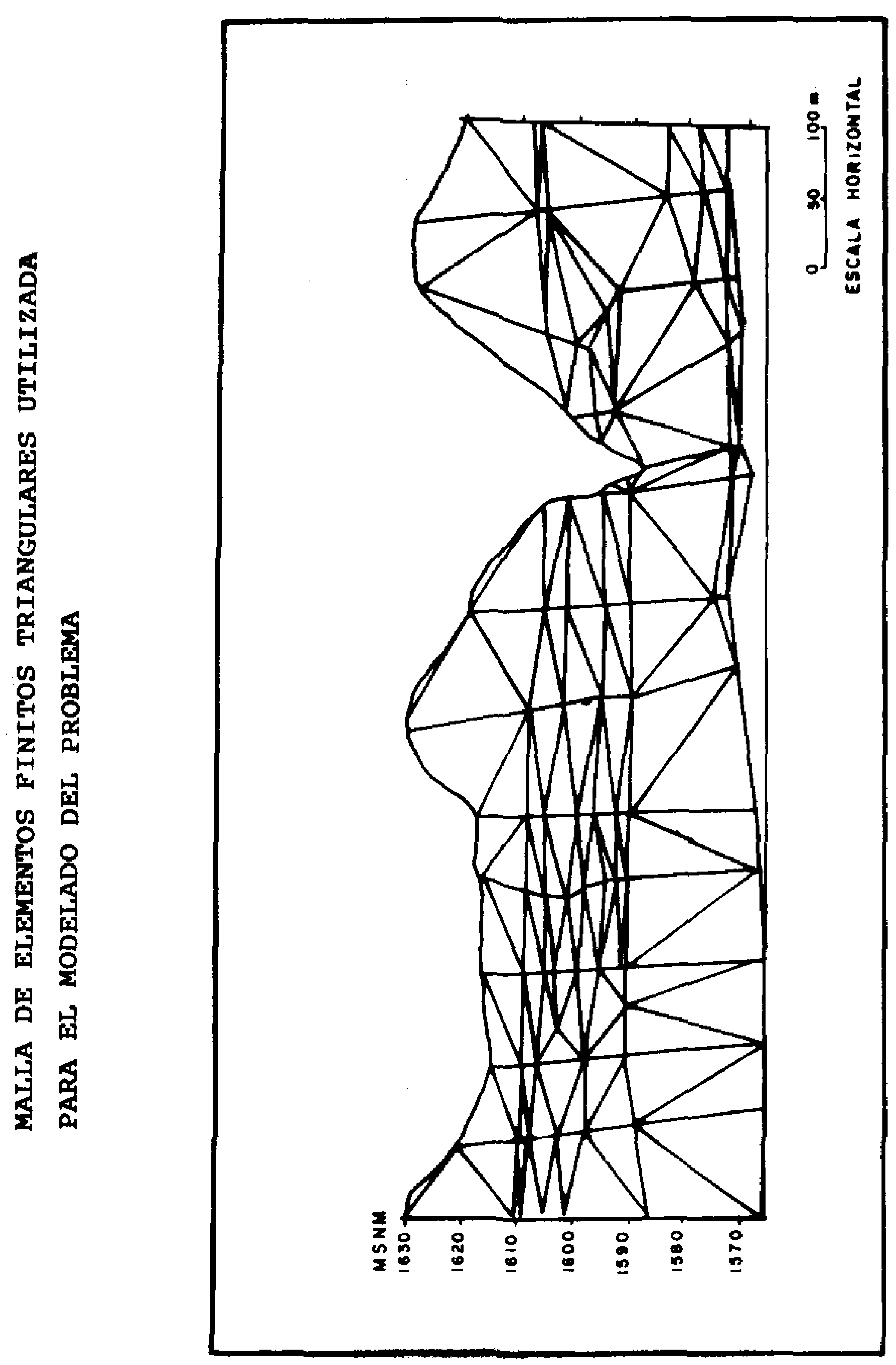

0
0
0
0 


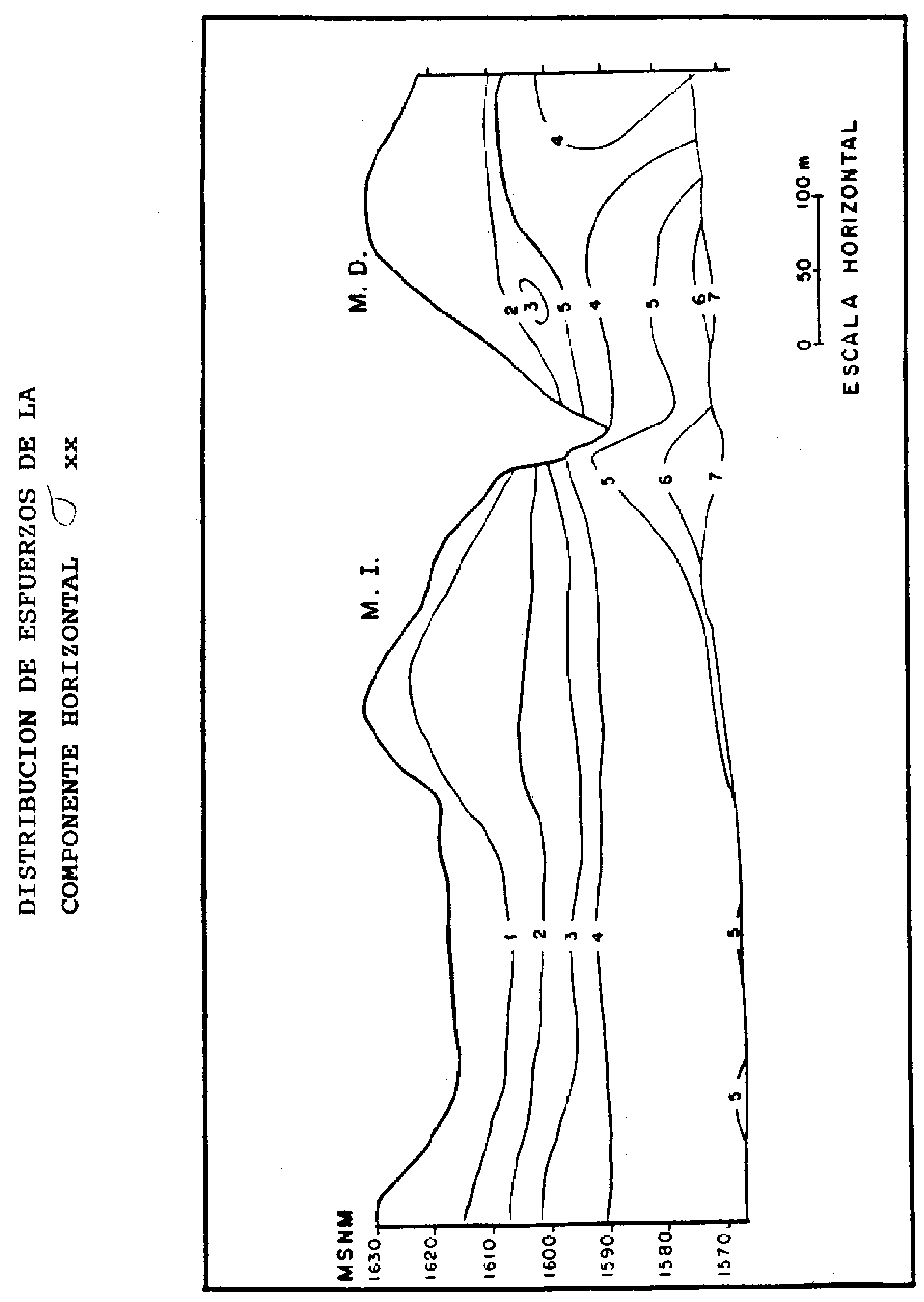

菖 


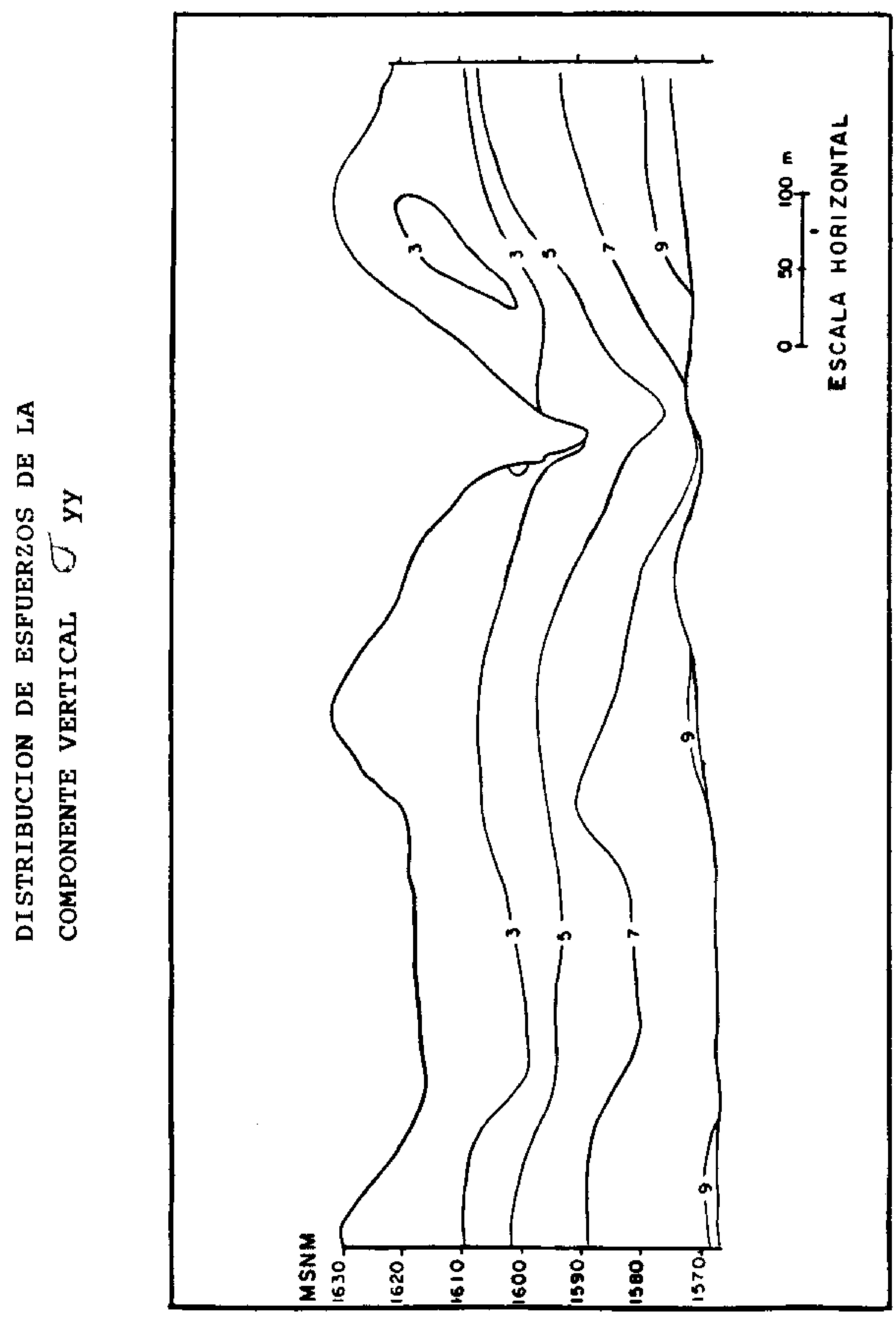

$\underset{0}{0}$ 
O5 dinas/cm que separa dos zonas de menor estabilldad a lo esperado. Esta discontinuidad está on la zona donde se cartografiaron los lentes de toba andesítica soldada intercalada en el horizonte arcilloso.

\section{CORTANTE (xy)}

La componente del cortante $(y x)$ es desde el punto de vista de inestabilidad, la más importante, ya que se asocian los altos valores absolutos con áreas de deslizamiento potencial y los gradientes altos de esta componente, se correlacionan con zonas inestables.

En la Figura 9, se observa que la parte central del valle del perfil del dique, se caracteriza por valores negativos con tendencia a desplazarse hacia la derecha, limitando dos zonas de comprensión definidas por las curvas del valor CERO, debidas al empuje gravitacional.

Los esfuerzos se concentran alrededor de la cañada; en esta zona y en la margen izquierda, las curvas están marcadas cada $50 \quad$ EO3 dinas/cm2.

Esta zona fue detectada en el perfil de la componente vertical.

La toba arcillo-arenosa de mala calidad presente en la margen derecha muestra una anomalía que define la zona inestable discutida en los perfiles de componentes vertical y horizontal. Esta inestabilidad es producto de la diferencia de capacidad de carga de los materiales de ambas márgenes.

En margen derecha se preveen reacomodos ligeros, mientras que en la margen izquierda se pueden suscitar desplazamientos repentinos, debido al gradiente de esfuerzos que en la margen izquierda cambla de 50 EO3 a 200 EO3 en menos de $20 \mathrm{~m}$. Por otro lado, en margen derecha se define una zona de deslizamiento en la curva de $50 \mathrm{EO} 3$ dinas $/ \mathrm{cm}$.

\section{CONCLUSIONES}

1) El método de elemento finito es de utilidad en clencias de la tierra, que para el caso expuesto, se usó para la zonificación de áreas inestables en un dique cortina.

2) El análisis de los perfiles de distribución de esfuerzos permitió identificar las áreas inestables, entre los cuales se destaca la zona del contacto entre la toba arcillo-arenosa y la arcilla, que fue definida en los tres perfiles $y$ previamente detectada por la geofísica.

3) En margen izquierda se observe un gradiente fuerte que implica una mayor concentración de esfuerzos cortantes de ese sitio.

4) Este método se puede aplicar a otros sistemas hidroeléctricos para complementar la información geotécnica del área.

\section{BIBLIOGRAFIA}

Aranda, L.J.R., 1985. Estudio, sísmico de "up-hole" en la Cortina Dique Talconapan, P.H. Atexcaco, Puebla. Informe Interno de CFE.

Cook, D. Robert, 1983. Concepts and aplications of Finite Element Analysis. John Wiley and Sons. new York. pp. 402. 


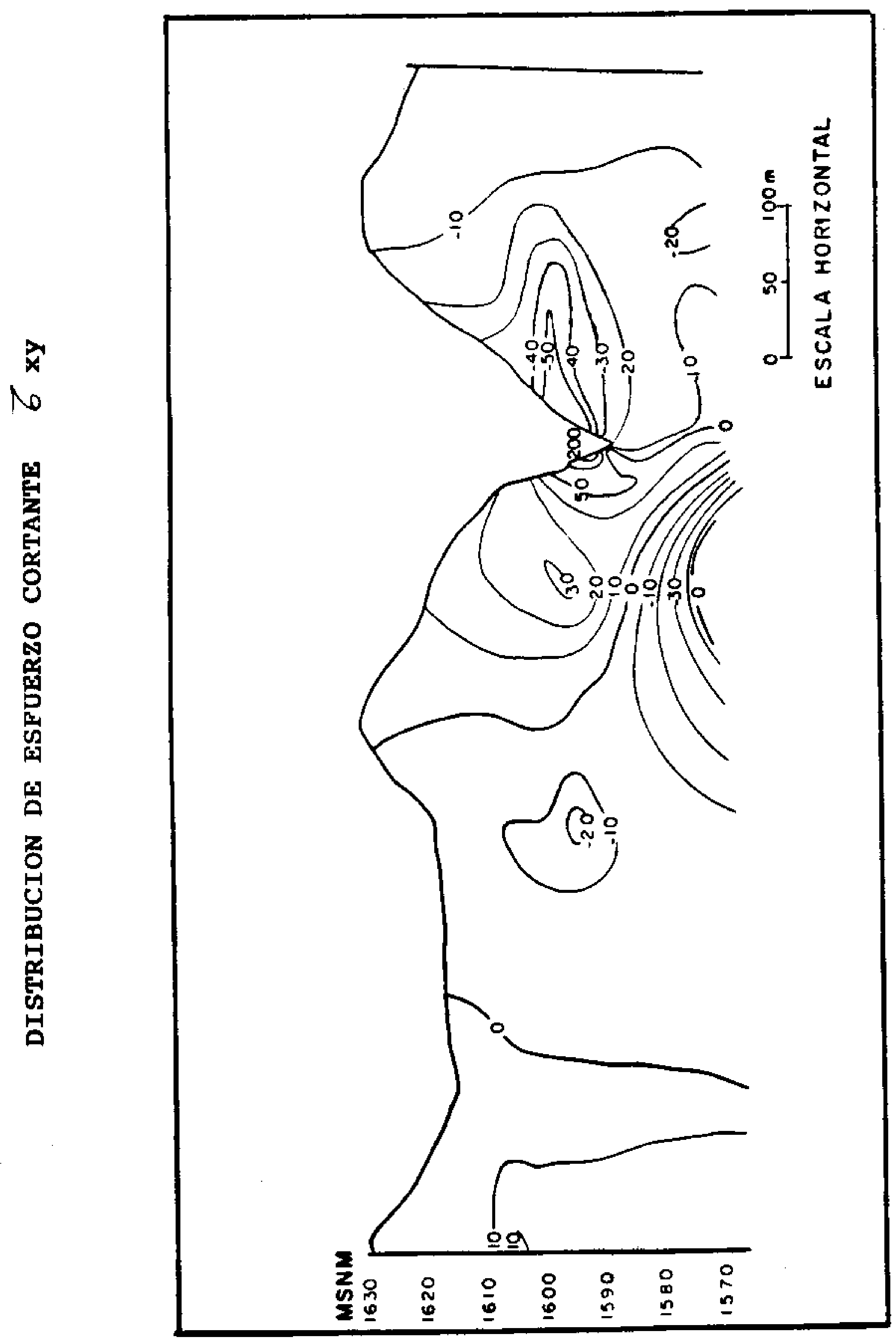

$\frac{9}{6}$ 
Devenport, G.C., Maldonado, Z.A. y Negrillo, M. Mexico. Pure and appl. Geophys. 120, 795-808. R., 1978. Técnicas Geofísicas para la Zienkiewicz, 0. C.; Cheung, Y. K. \& Stagg, K. G. Determinación de Propiedades Elásticas. 1966. Stress in Anisotropic Media with Particular Tecniterrae. 22 febrero-marzo.

Reference to Problema of Rock Uribe-Carbajal and Nyland, E. 1983; Elastic Mechanics.Journal of Stress Analysis, 1. 172-182. Models of Selsmic Failure in the Valley of 